\title{
Zadig: Une oeuvre rococo
}

\author{
Cherı Gish
}

Le style rococo s'est manifesté dans les domaines des arts, de la musique, et de la 1ittérature pendant à peu près tout le dix-huitième siècle. En ce qui concerne la littérature, 1a caractéristique la plus importante de ce style est que la thèse d'une oeuvre est implicite plutôt qu'explicite, comme dans le classicisme. Pour découvrir la thèse d'une telle oeuvre, le lecteur doit y participer. Une autre caractéristique importante du style rococo est la manière dans laquelle il incite cette participation: une tension est créée par le contraste entre la structure simple de l'oeuvre et ses nombreuses anecdotes et détails. Zadig, un des contes de Voltaire, est un bon exemple de ce style dit "rococo."

La structure de Zadig est très simple. E1le consiste en un mouvement linéaire d'épisode en épisode. Chaque épisode découle logiquement du précédent, pour qu'à la fin du conte il y ait une chaîne d'épisodes arrangés dans un ordre spécifique pour montrer le changement progressif de 1 'attitude de Zadig. Dans la première partie du conte Zadig croit que le bien est toujours récompensé, et il ignore le mal. Mais après quelques mauvaises expériences (la perte de deux femmes, l'exil de Babylone à cause de la jalousie du roi), il commence à douter de la vérité de son précepte et il découvre le mal. A la fin du conte Zadig croit encore que le bien est récompensé, mais il 
se rend compte de $I^{\prime}$ existence du mal, et du bien qu'on peut trouver dans le mal. La structure de cette oeuvre est évidemment linéaire, c'est-à-dire simple, comme celle du style rococo.

Dans toutes les anecdotes du conte on trouve beaucoup de détails exacts et, quelquefois, complexes. Ces détails donnent au lecteur beaucoup de renseignements sur les milieux géographiques, sur les apparences physiques des personnages, et sur les moeurs et les coûtumes de chaque pays dans lequel Zadig se trouve. Cette quantité de détails est nécessaire pour donner plus de vraisemblance à l'oeuvre, et pour stimuler et pour retenir 1'intérêt du lecteur. Plus $1^{\prime}$ intrique du conte s'avance, plus les détails se multiplient. Cette multiplication graduelle des détails constitue une sorte de défi intellectuel au lecteur: il doit découvrir la signification de tous les détails, la seule idée spécifique avec laquelle ils sont tous unis, comme sont les clefs en un porte-clefs. Pour réussir à trouver cette thèse qui est implicite dans l'oeuvre, le lecteur commence à participer (consciemment ou même inconsciemment) à 1'oeuvre. Cette participation exigée au lecteur constitue probablement 1 'aspect $1 e$ plus important du style rococo, c'est-à-dire la caractéristique qui le distingue nettement du style classique.

Enfin, il est évident que toutes les caractéristiques du style rococo se touvent dans Zadig. Donc, on peut dire que Zadig représente vraiment le style rococo, et qu'il en est aussi un très bon exemple.

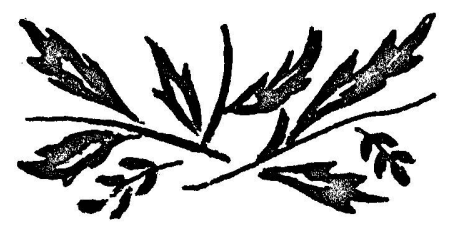

